\title{
Achieving a high dielectric tunability in strain-engineered tetragonal $\mathrm{K}_{0.5} \mathrm{Na}_{0.5} \mathrm{NbO}_{3}$ films
}

\author{
Lanxia Hao ${ }^{1,2}$, Yali Yang $\mathbb{D}^{3 凶}$, Yu Huan $\mathbb{D}^{4 凶}$, Hongbo Cheng ${ }^{2}$, Yu-Yao Zhao ${ }^{1}$, Yingying Wang ${ }^{1}$, Jing Yan ${ }^{1}$, Wei Ren $\mathbb{D}^{3}$ and \\ Jun Ouyang $\mathbb{1 D}^{2 \otimes}$
}

Using a modified Landau-Devonshire type thermodynamic potential, we show that dielectric tunability $\eta$ of a tetragonal ferroelectric film can be analytically solved. At a given electric field $E, \eta$ is a function of the remnant polarization $\left(P_{0}^{f}\right)$ and the smallfield relative dielectric permittivity $\left(x_{0}^{f}\right)$, which are commonly measured material properties. After a survey of materials, a large $\eta \sim 80 \%$ is predicted to be achievable in a (001)-oriented tetragonal $\left(\mathrm{K}_{0.5}, \mathrm{Na}_{0.5}\right) \mathrm{NbO}_{3}$ film. This strain-stabilized tetragonal phase is verified by density functional theory (DFT) calculations. $\left(\mathrm{K}_{0.5}, \mathrm{Na}_{0.5}\right) \mathrm{NbO}_{3}$ films based on this design were successfully prepared via a sputtering deposition process on $\mathrm{SrRuO}_{3}$-buffered $(100) \mathrm{SrTiO}_{3}$ substrates. The resulted epitaxial films showed a sizable $P_{0}^{f}(\sim 0.21 \mathrm{C}$ $\mathrm{m}^{-2}$ ) and a large $X_{0}^{f}(\sim 830-860)$, as well as a large $\eta$ close to the theoretical value. The measured dielectric tunabilities as functions of $E$ are well described by the theoretical $\eta(E)$ curves, validating our integrated approach rooted in a theoretical understanding.

npj Computational Materials (2021)7:62; https://doi.org/10.1038/s41524-021-00528-2

\section{INTRODUCTION}

Tunable dielectrics is a type of functional materials whose dielectric permittivity $(x)$ can be adjusted by an external electric field $E$. The key parameter used to describe a tunable dielectric is the so-called dielectric tunability, $\eta$, which is defined as the relative change $(\%)$ of dielectric permittivity under an $E$ field: $\eta(E)=\frac{x_{0}-X(E)}{x_{0}} \times 100 \%$. Here $X_{0}$ is the zero-field or small-field dielectric permittivity while $X(E)$ is the one under the field $E$. As a typical nonlinear dielectric, ferroelectrics are usually endowed with a high dielectric tunability, and hence have been broadly utilized in tunable dielectric devices. Recently, with the rapid developments in miniaturized and integrated tunable devices, such as phase shifters, oscillators, and filters, ferroelectric thin films showing a high $\eta$ value have been extensively investigated ${ }^{1-3}$. PZT and some lead-free films including $\mathrm{BaTiO}_{3}{ }^{4-6},\left(\mathrm{Ba}, \mathrm{Sr} \mathrm{TiO}_{3}{ }^{1,7-9}\right.$, and $\mathrm{Ba}(\mathrm{Zr}, \mathrm{Ti}) \mathrm{O}_{3}{ }^{10}$ are the center of focus based on their high dielectric permittivities. The reported $\eta$ values are usually between 20 and $70 \% 1,6,7,11,12$.

Currently, there are a few issues that need to be addressed for the further development of tunable ferroelectric dielectrics. Firstly, $\mathrm{BaTiO}_{3}$-based ferroelectrics have a relatively low Curie temperature $\left(<\sim 120^{\circ} \mathrm{C}\right)$ which has limited their applications, while the use of lead-based ferroelectrics (such as PZT) at elevated temperatures exacerbates the risk of toxic lead exposure. Secondly, the measured $\eta$ values $^{13-19}$ are usually not quantitatively explained, except for a few cases in bulk ceramics ${ }^{20,21}$. This is possibly due to the many practical factors affecting dielectric tunability in thin films, as well as disconnects between theorists and experimentalists. In this work, a coordinated effort integrating computational material design, material processing, and electrical characterization is reported for (001)-oriented epitaxial $\left(\mathrm{K}_{0.5} \mathrm{Na}_{0.5}\right) \mathrm{NbO}_{3}(\mathrm{KNN})$ films. As will be illustrated below, KNN in this structure is a promising material candidate for thin-film tunable dielectrics, owing to its lead-free composition, high Curie temperature, a sizable spontaneous polarization, and a large dielectric permittivity $^{22-25}$.

\section{RESULTS AND DISCUSSION \\ Dielectric tunability of a (001)-oriented tetragonal ferroelectric film}

For a (001)-oriented tetragonal ferroelectric film of an epitaxial quality, its polarization $P$ is aligned along the film normal under an

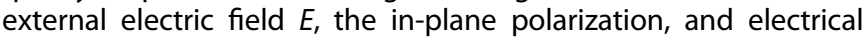
field components $P_{1}=P_{2}=0, E_{1}=E_{2}=0$, while the out-of-plane ones are not, $P_{3}=P \neq 0, E_{3}=E$. The in-plane strains extrapolated to the parent cubic phase, are $\varepsilon_{1}=\varepsilon_{2}=u_{m}=\varepsilon_{M}^{0}+Q_{12}\left(P_{0}^{b}\right)^{226}$, where $\varepsilon_{M}^{0}$ is the effective in-plane misfit, i.e., the difference between in-plane lattice parameters of the clamped and freestanding films, $Q_{12}$ is an electrostrictive coefficient, and $P_{0}^{b}$ is the spontaneous polarization of the free-standing film or bulk. The Landau-Devonshire type (LD) thermodynamic potential of the film can be expressed $a s^{26}$ :

$\tilde{G}=a_{1} P^{2}+a_{11} P^{4}+a_{111} P^{6}-E P+\frac{\left\{\varepsilon_{M}^{0}-Q_{12}\left[P^{2}-\left(P_{0}^{b}\right)^{2}\right]\right\}^{2}}{S}$

where $a_{1}, a_{11}, a_{111}$ are the first, second, and third-order Landau coefficients determined under the stress-free condition, and $S$ is the effective in-plane compliance given by $S=s_{11}+s_{12}$, with $s_{11}$, $s_{12}$ being the elastic compliances of the parent cubic phase. These material-specific parameters can be obtained from experimental measurements. By using the combo coefficients below ${ }^{26}$,

$$
a=2\left(a_{1}-\frac{2\left[\varepsilon_{M}^{0}+Q_{12}\left(P_{0}^{b}\right)^{2}\right] Q_{12}}{s}\right), \beta=4\left(a_{11}+\frac{Q_{12}^{2}}{S}\right), a_{3}=6 a_{111}
$$

\footnotetext{
${ }^{1}$ Key Laboratory for Liquid-Solid Structure Evolution and Processing of Materials (Ministry of Education), School of Materials Science and Engineering, Shandong University, Jinan, China. ${ }^{2}$ Institute of Advanced Energy Materials and Chemistry, Shandong Provincial Key Laboratory of Molecular Engineering, School of Chemistry and Chemical Engineering, Qilu University of Technology (Shandong Academy of Sciences), Jinan, China. ${ }^{3}$ International Centre for Quantum and Molecular Structures, Department of Physics, Shanghai

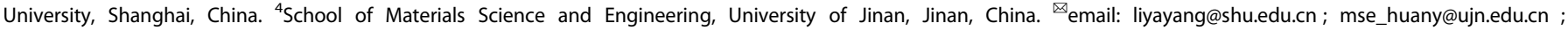
ouyangjun@qlu.edu.cn
} 
We reduce Eq. (1) into

$\tilde{G}=\frac{1}{2} a P^{2}+\frac{1}{4} \beta P^{4}+\frac{1}{6} a_{3} P^{6}-E P+\frac{\left[\varepsilon_{M}^{0}+Q_{12}\left(P_{0}^{b}\right)^{2}\right]^{2}}{S}$

The equilibrium polarization under an electric field $E$ can be obtained by minimizing the free energy in Eq. (3), i.e., $\mathrm{d} \tilde{G} / \mathrm{d} P=0$, which leads to

$E=a P+\beta P^{3}+a_{3} P^{5}$

when $E=0$, the film's remnant polarization can be obtained, $P_{0}^{f}=\sqrt{\frac{-\beta+\left(\beta^{2}-4 a a_{3}\right)^{1 / 2}}{2 a_{3}}}$. Using a field-induced polarization $p=P^{f}(E)-P_{0}^{f}$, the zero-field dielectric permittivity of the film $\left(X_{0}^{f}\right)$ can also be obtained from an approximation of Eq. (4), taking into account only the first term,

$E=\left[a+3 \beta\left(P_{0}^{f}\right)^{2}+5 a_{3}\left(P_{0}^{f}\right)^{4}\right] p$

$X_{0}^{f}=\left.\frac{\mathrm{dp}}{\mathrm{dE}}\right|_{E=0}=\left[a+3 \beta\left(P_{0}^{f}\right)^{2}+5 a_{3}\left(P_{0}^{f}\right)^{4}\right]^{-1}$

If the second-order term is taken into account, Eq. (4) reduces to $E=\gamma_{1} p+\gamma_{2} p^{2}$

where $\gamma_{1}=\left(x_{0}^{f}\right)^{-1}, \gamma_{2}=3 \beta P_{0}^{f}+10 a_{3}\left(P_{0}^{f}\right)^{3}$.

From the solution of Eq. (7) we can obtain the dielectric permittivity under an applied electric field $E$,

$X^{f}(E)=\frac{\mathrm{dp}}{\mathrm{dE}}=\frac{1}{\sqrt{\gamma_{1}^{2}+4 \gamma_{2} E}}=\frac{X_{0}^{f}}{\sqrt{1+\lambda_{f} E}}$

where $\lambda_{f}=\frac{4 \gamma_{2}}{\gamma_{1}^{2}}=4\left(x_{0}^{f}\right)^{2} \cdot\left[3 P_{0}^{f} \beta+10\left(P_{0}^{f}\right)^{3} a_{3}\right]$

It is noted that Eq. (8) reveals the nonlinear nature of the dielectric permittivity in a ferroelectric film. $\lambda_{f}$ is the tunability factor of the ferroelectric film, which characterizes the rate of the nonlinear change of its dielectric permittivity. Consequently, the dielectric tunability $\eta$ can be expressed as:

$\eta=\frac{X_{0}^{f}-X^{f}(E)}{X_{0}^{f}} \times 100 \%=1-\frac{1}{\sqrt{1+\lambda_{f} E}}$

From Eq. (10), it is clear that the dielectric tunability $\eta$ has a positive correlation with $\lambda_{f}$. The larger the $\lambda_{f}$, the higher the reduced portion of $\chi_{0}^{f}$, i.e., the higher the dielectric tunability $\eta$ is, at a given electric field E. From Eq. (9), $\lambda_{f}$ and hence $\eta$ is determined by the directly measurable material properties $X_{0}^{f}$ and $P_{0}^{f}$, given that the material-specific parameters of $a_{11}, a_{111}, S$ and $Q_{12}$ are known (In principle, all three parameters $\lambda_{f}, X_{0}^{f}$ and $P_{0}^{f}$ are functions of the misfit strain $\varepsilon_{M}^{0}$. They can be determined theoretically when the Landau coefficients $\left(a_{1}, a_{11}, a_{111}\right)$, the electrical/elastic/electromechanical properties $\left(P_{0}^{b}, \mathrm{~s}_{11} / \mathrm{s}_{12}\right.$ and $\left.Q_{12}\right)$ of the film material, as well as its misfit strain $\varepsilon_{M}^{0}$ are all correctly determined. However, this is not always possible for theorists or convenient for experimentalists. In this work, for the selection of material, we used the experimentally measurable properties of $x_{0}^{f}$ and $P_{0}^{f}$, which are abundant in the literature, and a reduced number of material-specific parameters).

In Fig. 1 below, the tunability factors $\lambda_{f}$ for some commonly used, (001)-oriented tetragonal ferroelectric films are presented as functions of $X_{0}^{f}$ and $P_{0}^{f}$. It clearly shows that, with an intermediate remnant polarization ( $P_{0}^{f} \sim 0.15$ to 0.3 ), a large dielectric permittivity (second only to PZT 50/50 in this chart, $X_{0}^{f} \sim$ $\left.600-1200^{18,25,27,28}\right)$, (001)-oriented tetragonal KNN films display a large $\lambda_{f}$ on the order of $10^{-6}-10^{-7}$, corresponding to a dielectric tunability $\eta \sim 59-86 \%$ under a typical electric field of $500 \mathrm{kV} \mathrm{cm}^{-1}$ ( $5 \mathrm{~V}$ on a $100 \mathrm{~nm}$ thick film). This is very promising for a lead-free ferroelectric.

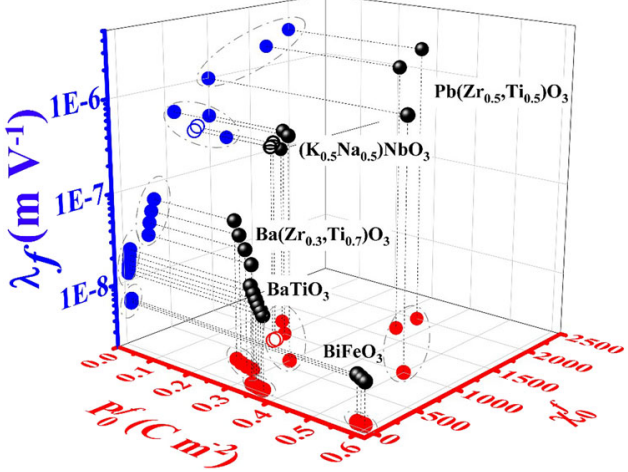

Fig. 1 Relationship between $\lambda_{\boldsymbol{f}}, X_{0}^{\boldsymbol{f}}$, and $\boldsymbol{P}_{0}^{\boldsymbol{f}}$. The relationship between $\lambda_{f}, X_{0}^{f}$ and $P_{0}^{f}$ of typical (001)-oriented tetragonal ferroelectric films, including $\mathrm{BaTiO}_{3}, \mathrm{Ba}\left(\mathrm{Zr}_{0.3}, \mathrm{Ti}_{0.7}\right) \mathrm{O}_{3}, \mathrm{BiFeO}_{3}$, $\mathrm{K}_{0.5} \mathrm{Na}_{0.5} \mathrm{NbO}_{3}$, and $\mathrm{Pb}\left(\mathrm{Zr}_{0.5} \mathrm{Ti}_{0.5}\right) \mathrm{O}_{3}$. The two open-circle points are experimental data from this work. The ellipses are drawn for the guidance of the eye.

Table 1. Parameters used for BTO, BZT 30/70, BFO, PZT 50/50 films in Fig. 1.

\begin{tabular}{llllll}
\hline Parameters & Units & BTO & BZT30/70 & BFO & PZT50/50 \\
\hline$a_{11}\left(10^{8}\right)$ & $\mathrm{C}^{-4} \mathrm{~m}^{6} \mathrm{~N}$ & -6.48 & 8.9 & 6.5 & 0.4764 \\
$a_{111}\left(10^{9}\right)$ & $\mathrm{C}^{-6} \mathrm{~m}^{10} \mathrm{~N}$ & 8 & -2.11 & 0.006 & 0.1336 \\
$\mathrm{~s}_{11}\left(10^{-12}\right)$ & $\mathrm{m}^{2} \mathrm{~N}^{-1}$ & 8.01 & 6.839 & 4.25 & 10.5 \\
$\mathrm{~s}_{12}\left(10^{-12}\right)$ & $\mathrm{m}^{2} \mathrm{~N}^{-1}$ & -1.66 & -2.093 & -4 & -3.7 \\
$\mathrm{Q}_{12}$ & $\mathrm{C}^{2} \mathrm{~m}^{4}$ & -0.045 & -0.0355 & -0.016 & -0.046 \\
$\beta\left(10^{9}\right)$ & $\mathrm{C}^{-4} \mathrm{~m}^{6} \mathrm{~N}$ & -1.32 & 4.26 & 7.94 & 1.44 \\
$a_{3}\left(10^{7}\right)$ & $\mathrm{C}^{-6} \mathrm{~m}^{10} \mathrm{~N}$ & 4802 & -1260 & 3.6 & 80.2 \\
References & & 29 & 30 & $31-33$ & 34 \\
\hline
\end{tabular}

The Landau coefficients and other related parameters of the ferroelectric films in Fig. 1 are listed in Tables 1 and 2, for $\mathrm{BaTiO}_{3}$ (BTO) $)^{29}, \mathrm{Ba}\left(\mathrm{Zr}_{0.3}, \mathrm{Ti}_{0.7}\right) \mathrm{O}_{3}(\mathrm{BZT} 30 / 70)^{30}, \mathrm{BiFeO}_{3}(\mathrm{BFO})^{31-33}$ and $\mathrm{Pb}$ $\left(\mathrm{Zr}_{0.5} \mathrm{Ti}_{0.5}\right) \mathrm{O}_{3}$ (PZT 50/50) ${ }^{34}$ films, and $\left(\mathrm{K}_{0.5} \mathrm{Na}_{0.5}\right) \mathrm{NbO}_{3}$ (KNN) films ${ }^{35}$, respectively.

\section{Strain-stabilized (001)-oriented tetragonal KNN film}

A modified-LD thermodynamic potential has been employed to predict the crystalline structure of an epitaxial KNN film ${ }^{35}$. The thermodynamic potential $\tilde{G}$ of a single-domain epitaxial film under a constant-stress mechanical boundary condition can be written as ${ }^{36-40}$ :

$$
\begin{aligned}
\tilde{G}= & a_{1}\left(P_{1}^{2}+P_{2}^{2}+P_{3}^{2}\right)+a_{11}\left(P_{1}^{4}+P_{2}^{4}+P_{3}^{4}\right)+a_{111}\left(P_{1}^{6}+P_{2}^{6}+P_{3}^{6}\right) \\
& +a_{12}\left(P_{1}^{2} P_{2}^{2}+P_{1}^{2} P_{3}^{2}+P_{2}^{2} P_{3}^{2}\right)+a_{123} P_{1}^{2} P_{2}^{2} P_{3}^{2} \\
& +a_{112}\left[P_{1}^{4}\left(P_{2}^{2}+P_{3}^{2}\right)+P_{3}^{4}\left(P_{1}^{2}+P_{2}^{2}\right)+P_{2}^{4}\left(P_{1}^{2}+P_{3}^{2}\right)\right] \\
& -\frac{1}{2} s_{11}\left(\sigma_{1}^{2}+\sigma_{2}^{2}+\sigma_{3}^{2}\right)-s_{12}\left(\sigma_{1} \sigma_{2}+\sigma_{1} \sigma_{3}+\sigma_{2} \sigma_{3}\right) \\
& -\frac{1}{2} s_{44}\left(\sigma_{4}^{2}+\sigma_{5}^{2}+\sigma_{6}^{2}\right)-Q_{11}\left(\sigma_{1} P_{1}^{2}+\sigma_{2} P_{2}^{2}+\sigma_{3} P_{3}^{2}\right) \\
& -Q_{12}\left[\sigma_{1}\left(P_{2}^{2}+P_{3}^{2}\right)+\sigma_{2}\left(P_{1}^{2}+P_{3}^{2}\right)+\sigma_{3}\left(P_{1}^{2}+P_{2}^{2}\right)\right] \\
& -Q_{44}\left(\sigma_{4} P_{2} P_{3}+\sigma_{5} P_{1} P_{3}+\sigma_{6} P_{1} P_{2}\right)-\left(P_{1} E_{1}+P_{2} E_{2}+P_{3} E_{3}\right)
\end{aligned}
$$

where $a_{1}, a_{11}, a_{12}, a_{111}, a_{112}$ and $a_{123}$ are the first and high-order Landau coefficients determined under the stress-free condition. $P_{i}$ $(i=1-3)$ and $\sigma_{j}(j=1-6)$ are the $i$ th polarization and $j$ th stress components, respectively. $s_{11}, s_{12}$, and $s_{44}$ are the elastic compliances of the parent cubic phase, while $Q_{11}, Q_{12}$, and $Q_{44}$ are the electrostrictive coefficients. 


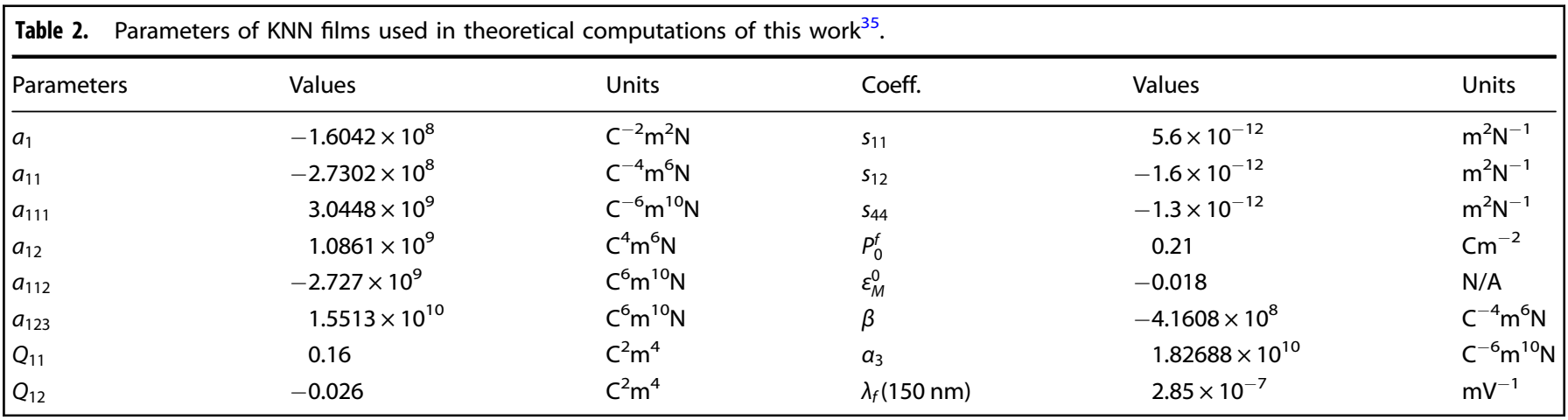

(a)

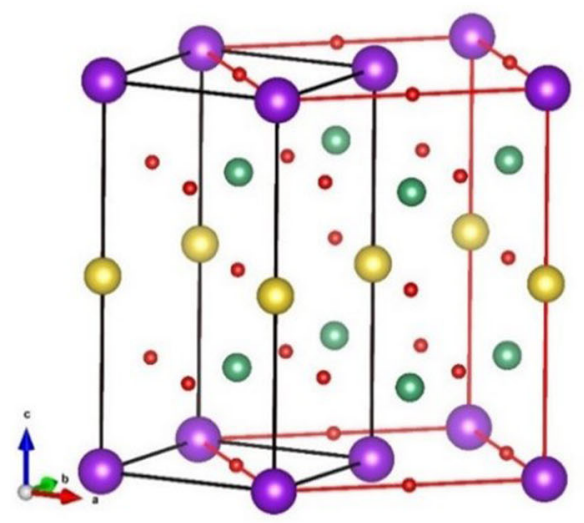

(b)

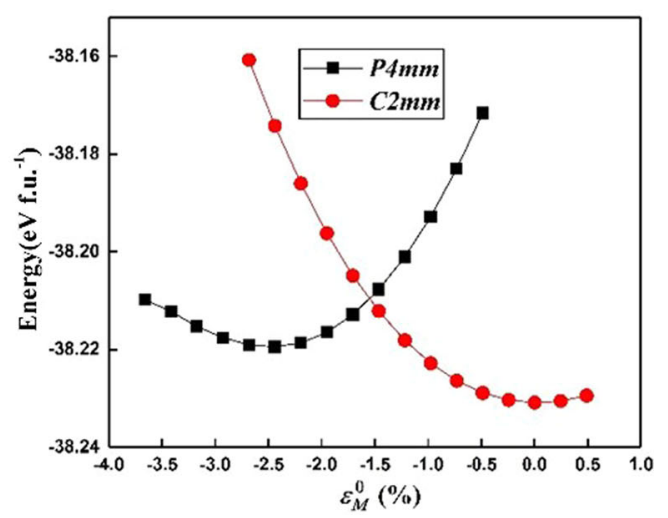

Fig. 2 Relevant results of DFT theoretical calculation. a Schematic diagram of ideal paraelectric KNN structure. The K/Na atom in the structure are ordered along [001] direction. The atoms in yellow, purple, green, and red are $\mathrm{K}, \mathrm{Na}, \mathrm{Nb}$, and $\mathrm{O}$, respectively. The unit cell in black frame and the supercell in red frame contains 10 and 20 atoms, respectively, and will be used in our calculation for different KNN phases, i.e., P4mm (10 atoms) and C2mm (20 atoms). b Free energies of the $P 4 \mathrm{~mm}$ and C2mm phases as functions of the misfit strain.

For an epitaxial oxide film, its in-plane strains decrease slowly with film thickness, after its initial relaxation via the formation of misfit dislocations in the first $10-50$ nm thick layer ${ }^{41,42}$. Therefore, for a few hundred nm thick or thicker oxide film, its in-plane strains are residual ones post relaxation, and can be considered as fixed/thickness-independent. Moreover, the stress components $\sigma_{3}$, $\sigma_{4}$, and $\sigma_{5}$ are zero because of the film's free surface. The relationship between the other in-plane strains $\varepsilon_{i}(i=1,2,6)$ and stress components can be obtained from the thermodynamic relations: $\frac{\partial G}{\partial \sigma_{1}}=-\varepsilon_{1}, \frac{\partial \tilde{G}}{\partial \sigma_{2}}=-\varepsilon_{2}, \frac{\partial \tilde{G}}{\partial \sigma_{6}}=-\varepsilon_{6}$.

For a (001) ferroelectric thin film epitaxially grown in its cubic paraelectric phase on a (100)-oriented cubic substrate, the inplane strains are $\varepsilon_{1}=\varepsilon_{2}=u_{m}=\varepsilon_{M_{37}}^{0}+Q_{12}\left(P_{0}^{b}\right)^{2}$ and $\varepsilon_{6}=0$. So, the thermodynamic potential becomes ${ }^{37}$ :

$$
\begin{aligned}
\tilde{G}= & a_{1}^{f}\left(P_{1}^{2}+P_{2}^{2}\right)+a_{3}^{f} P_{3}^{2}+a_{11}^{f}\left(P_{1}^{4}+P_{2}^{4}\right)+a_{33}^{f} P_{3}^{4}+a_{13}^{f}\left(P_{1}^{2} P_{3}^{2}+P_{2}^{2} P_{3}^{2}\right) \\
& +a_{12}^{f} P_{1}^{2} P_{2}^{2}+a_{111}\left(P_{1}^{6}+P_{2}^{6}+P_{3}^{6}\right)+a_{112}\left[\left(P_{2}^{2}+P_{3}^{2}\right) P_{1}^{4}+\left(P_{1}^{2}+P_{3}^{2}\right) P_{2}^{4}+\left(P_{1}^{2}+P_{2}^{2}\right) P_{3}^{4}\right] \\
& +a_{123} P_{1}^{2} P_{2}^{2} P_{3}^{2}+\frac{u_{m}^{2}}{s_{11}+s_{12}}-\left(P_{1} E_{1}+P_{2} E_{2}+P_{3} E_{3}\right)
\end{aligned}
$$

where $\quad a_{1}^{f}=a_{1}-\frac{\left(Q_{11}+Q_{12}\right) u_{m}}{s_{11}+s_{12}}, \quad a_{3}^{f}=a_{1}-\frac{Q_{12} u_{m}}{S_{11}+S_{12}}, \quad a_{11}^{f}=a_{11}+$ $\frac{s_{11}\left(Q_{11}^{2}+Q_{12}^{2}\right)-2 Q_{11} Q_{12} s_{12}}{2\left(s_{11}^{2}-s_{12}^{2}\right)}, a_{12}^{f}=a_{12}-\frac{s_{12}\left(Q_{11}^{2}+Q_{12}^{2}\right)-2 Q_{11} Q_{12} s_{11}}{s_{11}^{2}-s_{12}^{2}}+\frac{Q_{44}^{2}}{2 s_{44}} a_{13}^{f}=$ $a_{12}+\frac{\left(Q_{11}+Q_{12}\right) Q_{12}}{s_{11}+s_{12}}, a_{33}^{f}=a_{11}+\frac{Q_{12}^{2}}{s_{11}+s_{12}} \cdot a_{1}^{f}, a_{3}^{f}, a_{11}^{f}, a_{12}^{f}, a_{13}^{f}$ and $a_{33}^{f}$ are the modified Landau coefficients of the film. The equilibrium thermodynamic states can be determined via minimization of $\tilde{G}\left(\frac{\partial \tilde{G}}{\partial P_{1}}=0, \frac{\partial \tilde{G}}{\partial P_{2}}=0, \frac{\partial \tilde{G}}{\partial P_{3}}=0\right)$. For a KNN film, all the parameters used in the calculations are listed in Table 2. The KNN film is in a tetragonal phase at room temperature $\left(P_{1}=P_{2}=0, P_{3}=P \neq 0\right)$ when $\varepsilon_{M}^{0}$ is between -1.0 and $-2.0 \%$. This result is consistent with the theoretical works reported in the literature ${ }^{35,37}$. For undoped $\left(\mathrm{K}_{0.5} \mathrm{Na}_{0.5}\right) \mathrm{NbO}_{3}$ films grown on a commonly used perovskite-type semiconductor substrate $\left(\mathrm{SrTiO}_{3}, \mathrm{LaAlO}_{3},(\mathrm{La}, \mathrm{Sr})(\mathrm{Al}, \mathrm{Ta}) \mathrm{O}_{3}\right.$, etc.), this strain condition is readily achievable.

On the experimental side, there are several groups reporting strain- or composition-induced phases in $\left(\mathrm{K}_{x}, \mathrm{Na}_{1-x}\right) \mathrm{NbO}_{3}$-based epitaxial films ${ }^{43-46}$. For example, Luo et al. revealed a straininduced monoclinic phase in $\mathrm{Li}^{43}$ and $\mathrm{Mn}$-doped ${ }^{44} \mathrm{KNN}$ films, which shows an enhance piezoelectric response. Helden et al. ${ }^{45}$ revealed a linear correlation between the phase transition temperature from a strain-stabilized monoclinic phase to the orthorhombic $c$-phase in $\left(\mathrm{K}_{x}, \mathrm{Na}_{1-x}\right) \mathrm{NbO}_{3}$ films $(x=0.54-0.77)$, and the applied overall lattice strain from the sum of the anisotropic in-plane compressive misfit strains with the substrate. Shiraishi et al. ${ }^{46}$ showed a systematic reduction of the orthorhombic-tetragonal and tetragonal-cubic phase transition temperatures by increasing the Tadopant amount in thick epitaxial $(\mathrm{K}, \mathrm{Na})(\mathrm{Nb}, \mathrm{Ta}) \mathrm{O}_{3}$ films deposited by using the hydrothermal method. Together, these works provided the foundation for engineering the phase structures in KNN films.

It is noted that, unlike those of well-studied ferroelectrics, such as PZT or $\mathrm{BaTiO}_{3}$, the material parameters of KNN in its LD potential are not all determined from experimental measurements ${ }^{35}$. To verify this material design, we used density functional theory (DFT) calculations $^{47-49}$ to investigate the relative stability between the bulk orthorhombic and the strain-induced tetragonal phases.

As shown in Fig. 2a, the supercells with $\mathrm{K}$ and $\mathrm{Na}$ atoms orderly distributed along the [001] direction are adopted in our 
(a)

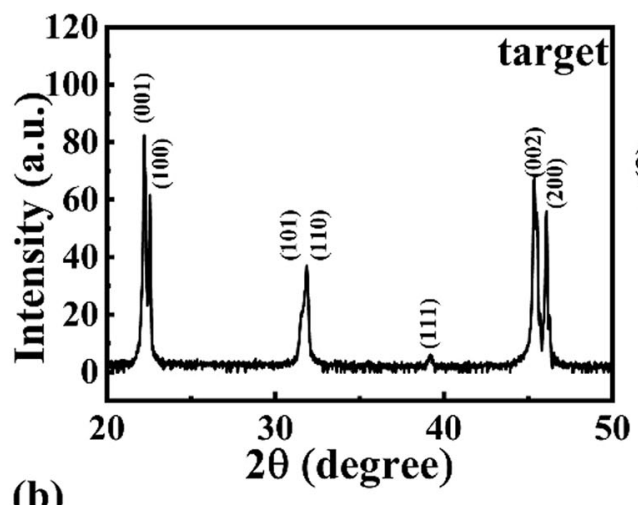

(b)

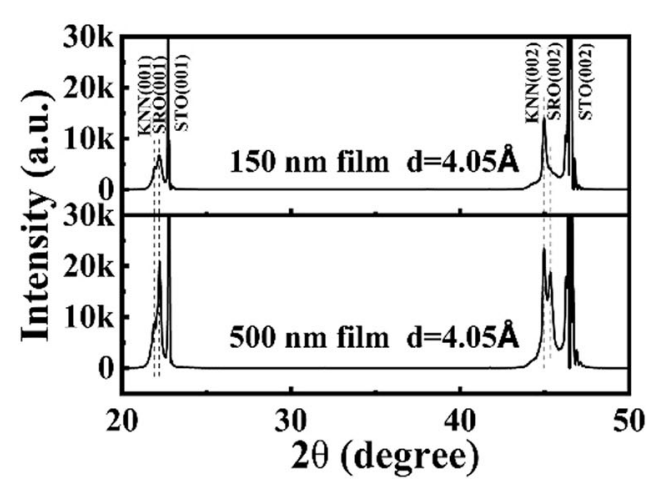

(c)

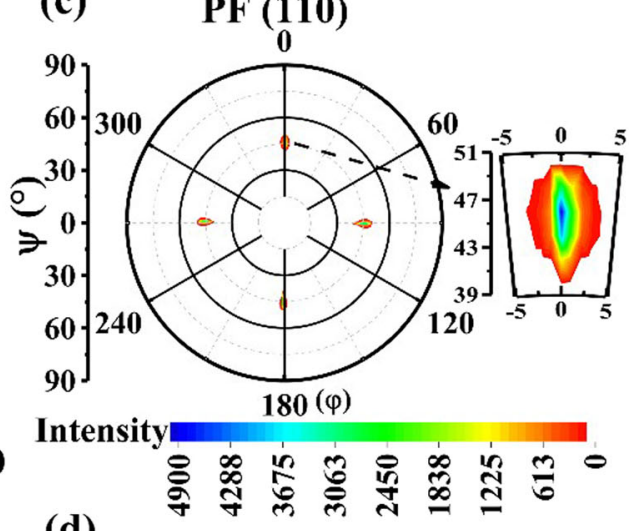

(d)

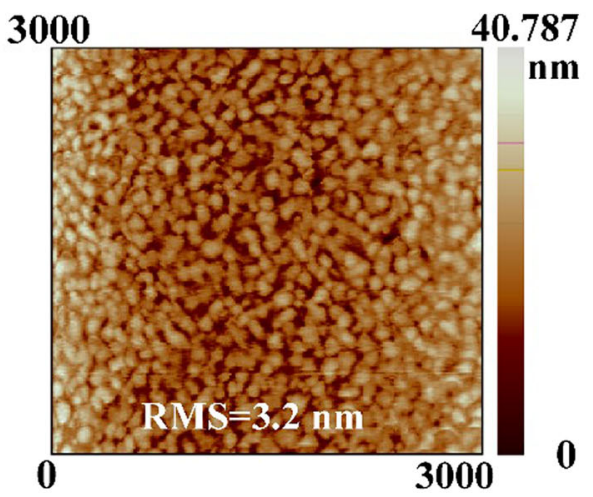

Fig. 3 Microstructure and morphology of KNN target and films. X-ray diffraction $2 \theta$ scan patterns of (a) the KNN ceramic target and (b) $150 \mathrm{~nm}$ - and $500 \mathrm{~nm}$-thick KNN films; c the (110) XRD pole figure of the $500 \mathrm{~nm}$ KNN film; d an AFM surface scan image of the $150 \mathrm{~nm}$ KNN film.

simulation. Moreover, two different phases, i.e., tetragonal $P 4 \mathrm{~mm}$ and orthorhombic $\mathrm{C} 2 \mathrm{~mm}$ which contain 10 and 20 atoms, respectively, are considered for their relative stabilities under a biaxial strain. The misfit strain of the KNN structure can be represented by: $\varepsilon_{M}^{0}(\%)=\frac{a-a_{0}}{a_{0}} \times 100 \%$, where $a_{0}$ is the in-plane lattice parameter of the bulk stable phase of $c 2 \mathrm{~mm}$. The free energy-misfit strain diagram for the two phases is shown in Fig. 2b. It clearly shows that a compressive strain will destabilize the bulk $C 2 \mathrm{~mm}$ phase, and induce the tetragonal $P 4 \mathrm{~mm}$ phase. The transition point is at $\varepsilon_{M}^{0} \sim-1.5 \%$, fairly consistent with the prediction based on the LD thermodynamic computation ${ }^{35,37}$.

\section{Analysis of microstructure and chemical states of the KNN films}

Based on the above analysis, we designed and obtained (001)oriented tetragonal KNN films (150 and $500 \mathrm{~nm}$ thick) via RF magnetron sputtering on $\mathrm{SrRuO}_{3}$ (SRO)-buffered (100) $\mathrm{SrTiO}_{3}$ (STO) substrates, which showed a high tunability factor $\lambda_{f} \sim 3 \times$ $10^{-7}$, and a large dielectric tunability $\sim 80 \%$.

Figure 3 shows the microstructural characteristics of the KNN target and films. In Fig. 3a, except for the XRD peaks corresponding to a bulk orthorhombic phase of $\mathrm{KNN} 50 / 50^{50}$, no other secondary phases were detected in the KNN ceramic target. The lattice parameters of the target are $c_{\mathrm{pc}}=a_{\mathrm{pc}}=3.997 \AA, b_{\mathrm{pc}}=$ $3.938 \AA$, respectively. In Fig. $3 b$, both KNN films show a $(00 /)$ orientation with an out-of-plane lattice parameter of $\sim 4.05 \AA$. Furthermore, in the (110) pole figure (Fig. 3c), the diffracted X-ray signals display a fourfold symmetry at a tilt angle of $\sim 45^{\circ}$, indicating a heteroepitaxial growth of the KNN film with a (00/) out-of-plane orientation ${ }^{51}$. It will be revealed later, that these films have a tetragonal symmetry with a $(00 /)$-orientation promoted by the SRO buffer layer, which lowered the surface energy of the $(00 /)$ crystalline plane. The $4.05 \AA$ out-of-plane lattice parameter corresponds to an in-plane compressive strain of $\sim-1.8 \%$ $\left(\varepsilon_{x x}=\varepsilon_{y y}=-\frac{1-v}{2 v} \varepsilon_{z z}, \varepsilon_{z z}\right.$ is the out-of-plane strain, $\varepsilon_{z z}=\frac{c_{\text {film }}-c_{\text {bulk }}}{c_{\text {bulk }}}$, $c_{\text {film }}$ and $c_{\text {bulk }}$ are the out-of-plane lattice parameters of the KNN film and bulk ceramic, $v$ is the Poisson's ratio, which is $\sim 0.27$ for $\mathrm{KNN}^{52}$ ). This compressive strain is responsible for the elongation of the out-of-plane lattice parameter and shortening of the in-plane $a$ and $b$ lattice parameters. Lastly, a representative AFM surface scan image of the $150 \mathrm{~nm}$ KNN film is shown in Fig. 3d. The film is dense and uniform, displaying a smooth surface with a root-meansquare (RMS) roughness of $\sim 3.2 \mathrm{~nm}$.

Figure $4 \mathrm{a}$ and e are representative cross-sectional bright-field TEM images of the $150 \mathrm{~nm}$ and $500 \mathrm{~nm}$ KNN film, respectively, which reveal clean and sharp interfaces in the KNN/SRO/STO heterostructure. The thicknesses of the SRO layer in both films were $\sim 60 \mathrm{~nm}$. In the high-resolution TEM images shown in Fig. 4b, $\mathrm{c}, \mathrm{f}$, and $\mathrm{g}$, atomically coherent interfaces (marked by dashed white lines) between the (100) STO substrate and the SRO layer, SRO and KNN layers were revealed, validating a $(00 /)$ heteroepitaxial growth in the two films. Figure $4 d$ is a selected area electron diffraction (SAED) pattern of the boxed region in Fig. $4 c$ via a fast-Fourier transformation (FFT), while Fig. $4 \mathrm{~h}$ is a SAED pattern directly taken from the circled region in Fig. 4e. The zone axis is [010] for both SAED patterns. The measured out-of-plane and in-plane lattice parameters of the KNN films were $\sim 4.05 \AA$ and $\sim 3.93 \AA$, respectively. The sharp electron diffraction spots in Fig. $4 d$ and $h$ indicate that the films are of epitaxial quality, consistent with the XRD results displayed in Fig. 3.

Surface XPS spectra of the $150 \mathrm{~nm}$ KNN film were collected to analyze the valence states of the elements. The core-level 


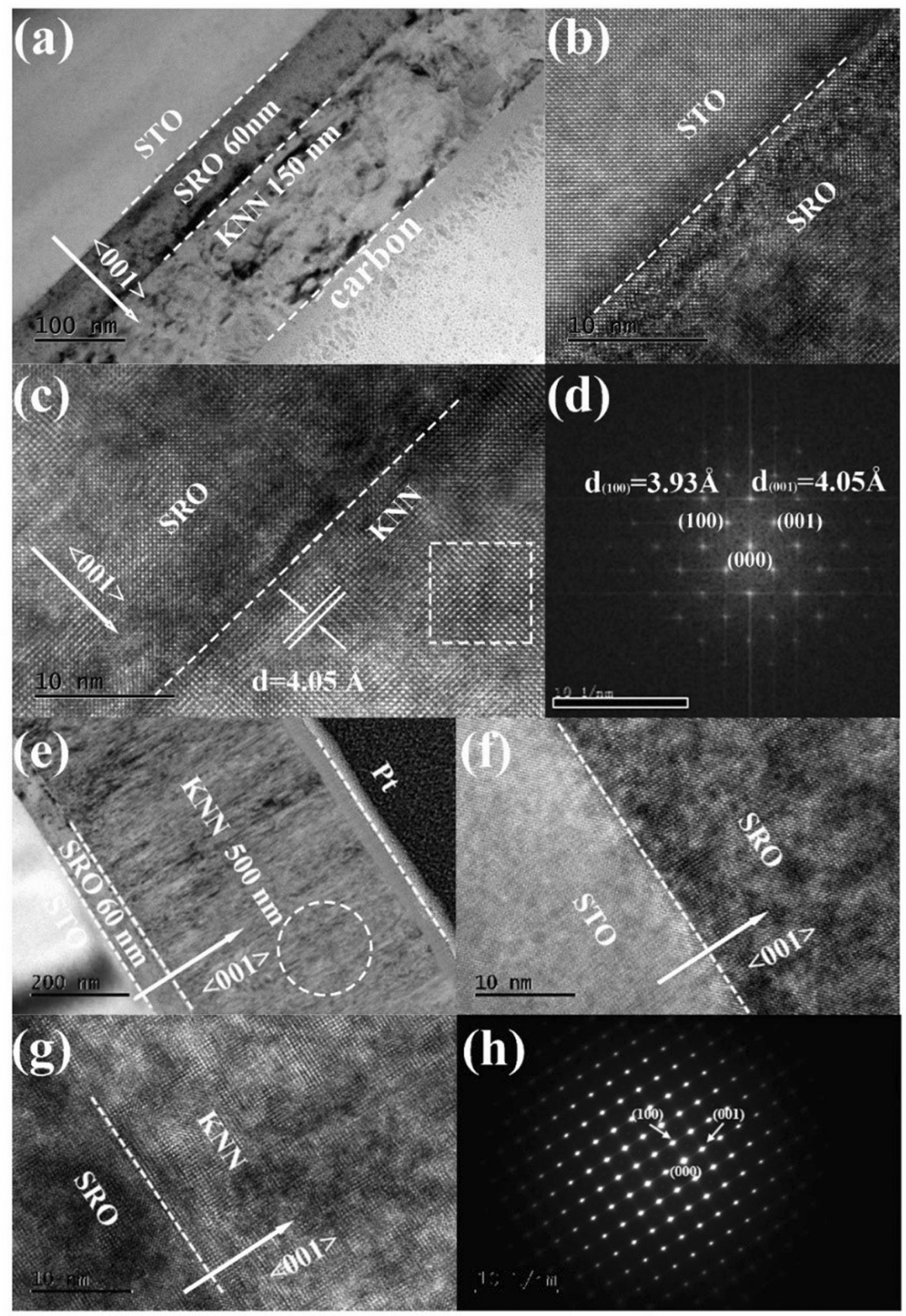

Fig. 4 Cross-sectional microstructure of KNN films. Cross-sectional bright-field TEM images of the KNN/SRO/STO heterostructure with the KNN thickness of (a) $150 \mathrm{~nm}$ and (e) $500 \mathrm{~nm}$; b-c and $\mathbf{f}-\mathbf{g}$ are the high-resolution TEM images near the SRO/STO and KNN/SRO interfaces for the $150 \mathrm{~nm}$ and $500 \mathrm{~nm}$ thick KNN films, respectively; $\mathbf{d}$ and $\mathbf{h}$ are the selected area electron diffraction patterns of the boxed and circled regions in (c) and (e), respectively.

elemental binding energies were all calibrated by using the carbon $1 s$ peak $(284.8 \mathrm{eV})$, and the XPS spectra were fitted by using the Avantage software. Figure $5 \mathrm{a}$ is the survey spectrum which showed clear signals from $\mathrm{K}, \mathrm{Na}, \mathrm{Nb}$, and $\mathrm{O}$, as well as a trace amount of the environment $\mathrm{C}$. No fingerprints of any other element were found in the survey spectrum, which proved the chemical purity of the KNN film. Figure $5 \mathrm{~b}$ is the core level $\mathrm{K} 2 p$
XPS spectrum. The K $2 p$ XPS peak from the $\mathrm{K}$ element in the KNN lattice splits into doublets $\left(\mathrm{K} 2 p_{3 / 2} \text { and } \mathrm{K} 2 p_{1 / 2}\right)^{53-57}$. There is a very weak second group of doublets, marked as K $2 p_{3 / 2}^{\prime}$ and $\mathrm{K} 2 p_{1 / 2}^{\prime}$, which can be attributed to a small amount of $K$ existing in the form of $\mathrm{K}_{2} \mathrm{O}^{53-57}$. The existence of potassium oxide on the film surface is usually unavoidable ${ }^{53-57}$, which can be attributed to the effects of composition variation and different evaporation rates of 
(a)

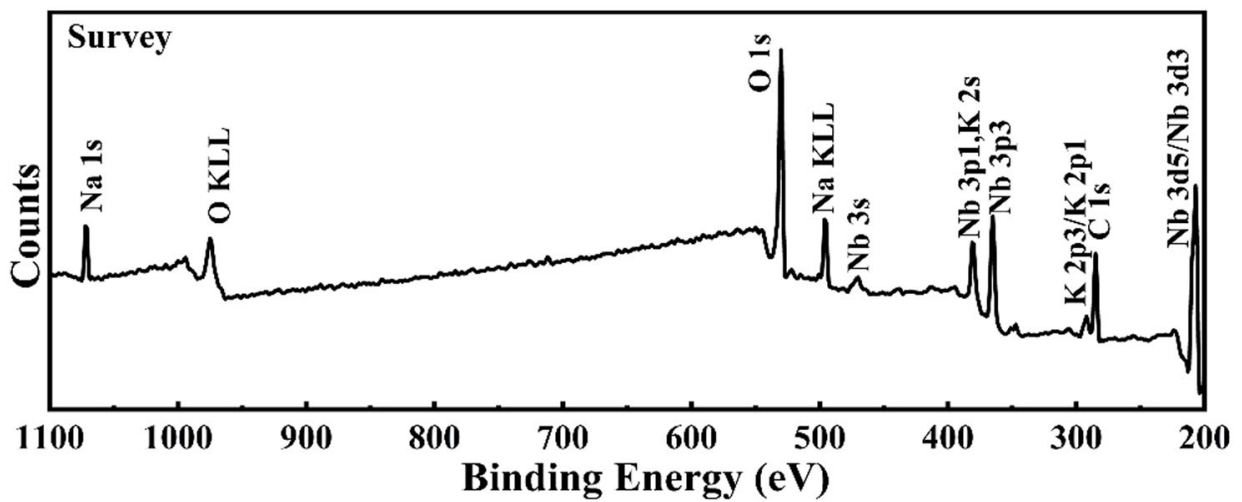

(b)

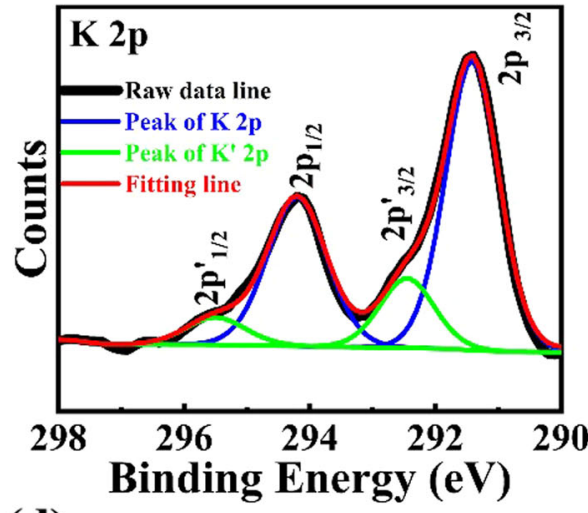

(c)

(d)

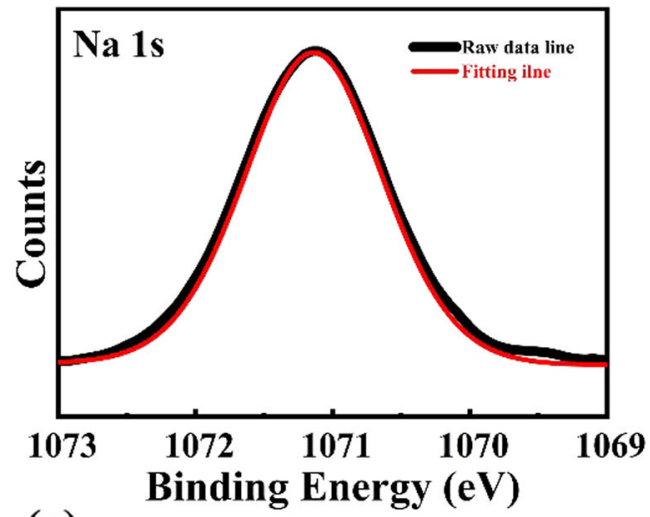

(e)
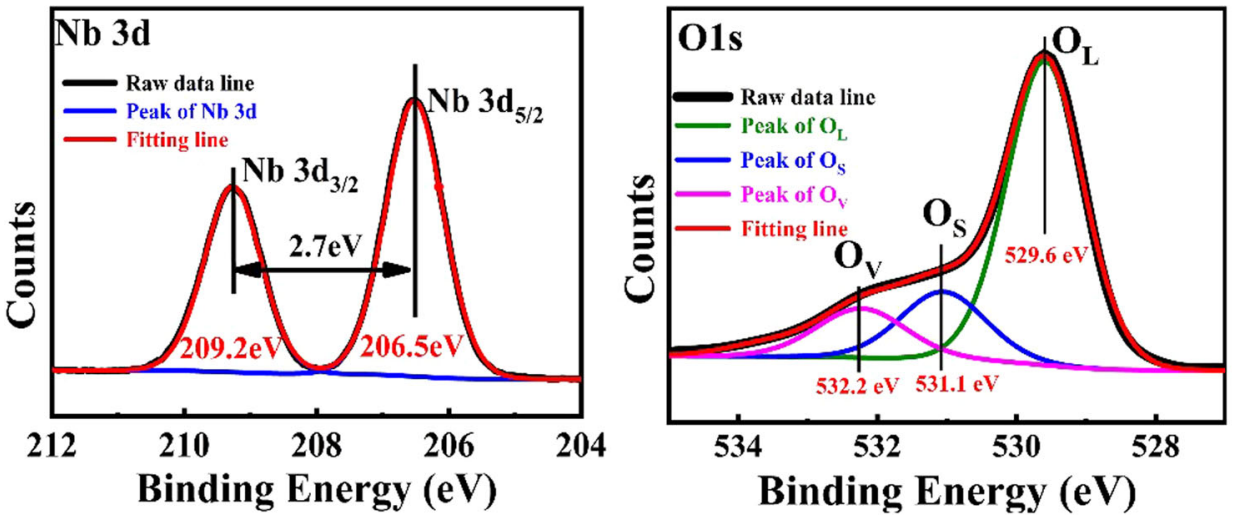

Fig. 5 Chemical properties of the KNN films. a survey spectrum and (b-e) narrow-scan spectra of (b) K 2p, c Na 1s, d Nb $3 d$, and (e) O 1s core levels from the $150 \mathrm{~nm}$ KNN film.

the component species in the KNN target and film. Furthermore, the $1 s$ core-level XPS spectrum of $\mathrm{Na}$ and the $3 d$ core-level XPS spectrum of $\mathrm{Nb}$ are shown in Fig. $5 \mathrm{c}$ and d, respectively. The $\mathrm{Na} 1 \mathrm{~s}$ peak is symmetrical and shows no split or shift of peak position. It is well fitted to the standard peak for the sodium element in the KNN lattice. On the other hand, the $\mathrm{Nb}$ peak splits into doubles $\left(3 d_{3 / 2}\right.$ and $\left.3 d_{5 / 2}\right)$ with a binding energy difference of $\sim 2.7 \mathrm{eV}$, confirming the existence of $\mathrm{Nb}-\mathrm{O}$ bonds and a $\mathrm{Nb}^{5+}$ valence state in the film ${ }^{58}$. Figure $5 \mathrm{e}$ is the $\mathrm{O} 1 \mathrm{~s}$ core-level XPS spectrum, which is asymmetric and can be decomposed into three characteristic peaks, indicating the existence of three oxygen species on the film surface. The peak located at the low energy side $(529.6 \mathrm{eV})$ is associated with the lattice oxygen $\left(\mathrm{O}_{\mathrm{L}}\right)$. The middle peak, located at $531.1 \mathrm{eV}$, corresponds to adsorbed oxygen on the surface $\left(\mathrm{O}_{S}\right)$, which may have a variety of states, such as $\mathrm{O}^{-}, \mathrm{O}_{2}{ }^{2-}$, etc. Lastly, the peak located at $532.2 \mathrm{eV}$ is associated with oxygen vacancies $\left(\mathrm{O}_{\mathrm{v}}\right)^{59}$. The formation of oxygen vacancies on the surface of a KNN film is commonly observed ${ }^{60,61}$, which can be attributed to the evaporation loss of volatile alkali ions ${ }^{55}$.

\section{Electrical performance}

Figure 6a shows the measured $P-E$ hysteresis loops of the two KNN films (@1 kHz). Both films display a remnant polarization of $\sim 21 \mu \mathrm{Ccm}^{-2}$. In Fig. 6b, the measured dielectric permittivity $X^{-}$ electric field $E(X-E)$ curve of the $500 \mathrm{~nm}$ film is well fitted by the theoretical results given by Eqs. (8) and (9), while that of the $150 \mathrm{~nm}$ film was offset from the computed $X-E$ curve by a small field/voltage $(\sim 0.225 \mathrm{~V}$, inset of Fig. $6 \mathrm{~b})$. This small offset can be attributed to a non-negligible voltage drop across an interface 
(a)

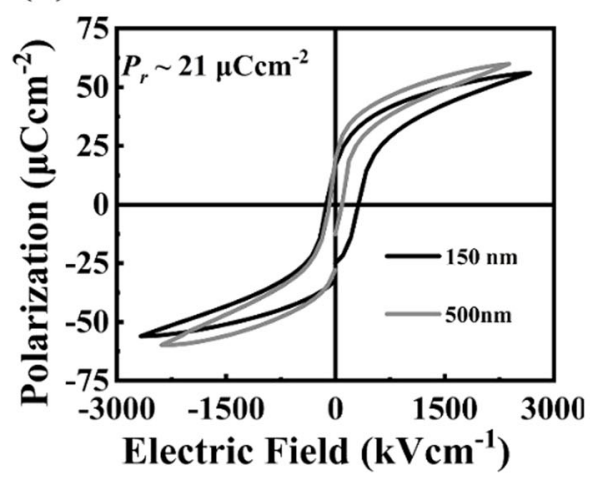

(c)

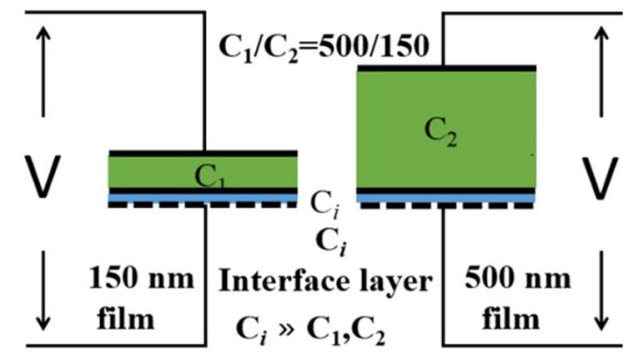

(b)

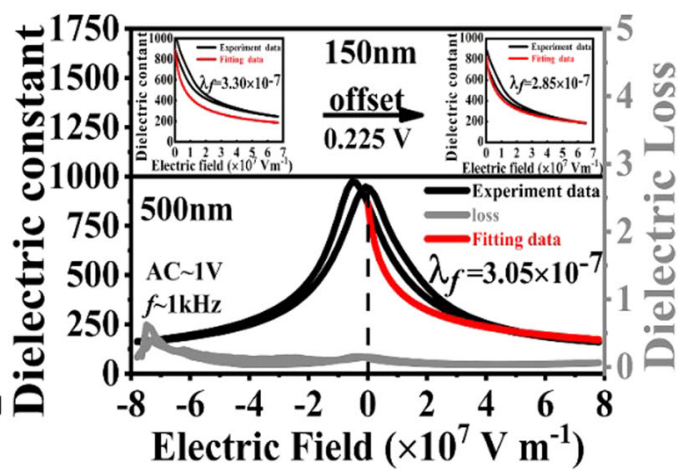

(d)

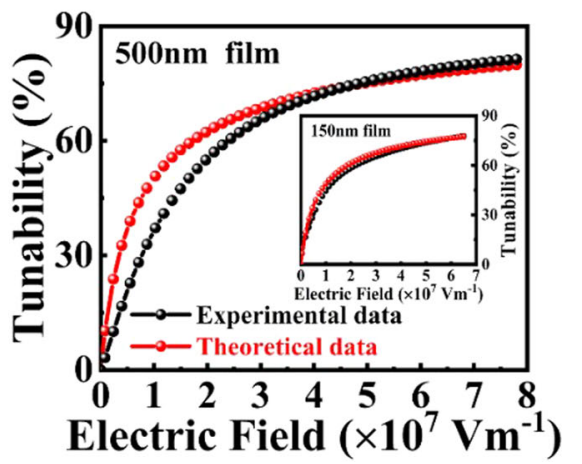

Fig. 6 Electrical performance of the KNN films. a Polarization-electric field $(P-E)$ hysteresis loops (measured @1 kHz); b measured and computed dielectric permittivity $\chi$-electric field $E(\chi-E)$ curves of the $500 \mathrm{~nm}$-thick KNN film $\left(\lambda_{f}=3.05 \times 10^{-7} \mathrm{~m} \mathrm{~V} \mathrm{~V}^{-1}\right.$ from Eq. 9), the inset shows the measured and computed $\chi-E$ curves $(E>0)$ of the $150 \mathrm{~nm}$-thick film under $10 \mathrm{~V}\left(\lambda_{f}=2.85 \times 10^{-7} \mathrm{~m} \mathrm{~V}^{-1}\right.$ from Eq. 9). The dielectric loss tangent-electric field $(\operatorname{tg} \delta-E)$ curves are also shown for the $500 \mathrm{~nm}$ film. c Schematic illustration of the effect of a capacitive interface layer on reducing the effective voltage across the KNN film. The thinner the KNN film, the larger the voltage drop on this interface layer. $\mathbf{d}$ Measured (@1 kHz) and computed dielectric tunabilities as functions of the bias field for the 500 and $150 \mathrm{~nm}$ (inset) KNN films.

capacitive layer. The effect of such an interface layer is schematically shown in Fig. 6c. A simple model of serially connected capacitors is used to illustrate the effect of film thickness on the voltage drop across a thin interface layer ${ }^{62}$. When the KNN film is thick enough, its capacitance becomes very low compared with that of the interface layer, and hence nearly $100 \%$ of the applied voltage drops across the KNN film. The interfacial voltage drop only becomes non-negligible when the film is much thinner ( $500 \mathrm{~nm} \rightarrow 150 \mathrm{~nm}$ ). After correction of this small offset, it is noted that there is only a small difference between the computed tunability factors $\lambda_{f}$ (via Eq. (9)) of the two films, $3.05 \times$ $10^{-7} \mathrm{~m} \mathrm{~V}^{-1}$ for the $500 \mathrm{~nm}$ film and $2.85 \times 10^{-7} \mathrm{~m} \mathrm{~V}^{-1}$ for the $150 \mathrm{~nm}$ one, which can be attributed to their slightly different remnant dielectric permittivities $\left(X_{0}^{f} \sim 860\right.$ and $\sim 830$ for the $500 \mathrm{~nm}$ and $150 \mathrm{~nm}$ KNN films, respectively). In addition, the dielectric loss tangent-electric field ( $\operatorname{tg} \delta$-E) curve is also shown for the $500 \mathrm{~nm}$ film. The losses are in a reasonable range $(\sim 0.03$ to 0.1) except at the high negative bias end. This is possibly due to the asymmetric electrode interfaces ${ }^{62}$. Lastly, Fig. $6 \mathrm{~d}$ displays the experimental and computed dielectric tunability-electric field $(\eta-E)$ curves for the two KNN films. The experimental curves were derived from the $x-E$ data in Fig. $6 b$, while the theoretical curves were computed by using Eqs. (9) and (10), with a $0.225 \mathrm{~V}$ voltage offset correction performed for the $150 \mathrm{~nm}$ film. The experimental and theoretical dielectric tunabilities agreed fairly well with each other, especially in the high electric field end, where both curves saturate with an increasing field. The small gap between the experimental tunability and the theoretical one, which exists in the low-to-middle field range, can be attributed to charged defects in the films, which demand an extra amount of energy to be aligned with the applied electric field. Effects of these extrinsic charges diminish under a high electric field, hence the measured tunabilities (and dielectric permittivities as shown in Fig. 6b) behaved just like the theory predicted in the high field end. Number-wise, the designed high tunability values have been achieved in the KNN films. For the $150 \mathrm{~nm}$-thick KNN film, the theoretical tunability $\eta$ under an electric field of $6.5 \times 10^{7}\left(\mathrm{~V} \mathrm{~m}^{-1}\right)(10 \mathrm{~V})$ is $77.4 \%$, while the measured $\eta$ is $77.7 \%$. For the 500 nm-thick KNN film, the computed and measured $\eta$ values at $7.8 \times 10^{7}\left(\mathrm{~V} \mathrm{~m}^{-1}\right)(39 \mathrm{~V})$ were $80 \%$ and $81 \%$, respectively. These numbers are the highest reported $\eta$ values so far for KNN thin film dielectrics ${ }^{17-19,25,63}$.

In this work, (001)-oriented, strain-stabilized tetragonal KNN thin films with a high dielectric tunability were successfully designed via computational approaches using the LD type thermodynamic potential and DFT calculations. These KNN films were epitaxially grown on $\mathrm{SrRuO}_{3}$-buffered (100) $\mathrm{SrTiO}_{3}$ substrates via $\mathrm{RF}$ magnetron sputtering. A high remnant polarization $P_{0}^{f}(\sim 21 \mu \mathrm{C}$ $\left.\mathrm{cm}^{-2}\right)$ and a large small-field dielectric permittivity $X_{0}^{f}(\sim 830-860)$ were achieved in these films. The measured maximum dielectric tunabilities $\eta(\sim 75-80 \%)$ are in good agreement with the computed theoretical values. Moreover, their field dependences, i.e., the $\eta$ - $E$ curves, can be well described by our equation derived using the LD theory. These results suggest that, the dielectric tunability of a ferroelectric film, as its key performance parameter in tunable dielectrics, can be predicted using a computational approach. 


\section{METHODS \\ DFT calculations}

The DFT calculations were performed with the projector augmented wave (PAW) approach ${ }^{47}$, as implemented in the Vienna ab initio simulation package $(\mathrm{VASP})^{48}$. The electron exchange-correlation functional was treated by generalized gradient approximation (GGA) in the form of Perdew-Burke-Ernzerhof (PBE) ${ }^{49}$. The plane-wave cutoff and convergence criteria for energy and force are set to be $550 \mathrm{eV}, 10^{-7} \mathrm{eV}$, and $0.01 \mathrm{eV} \AA^{-1}$, respectively.

\section{Sintering of the KNN ceramic target}

The KNN 50/50 ceramic target was prepared by using a conventional solidstate reaction process. $\mathrm{Na}_{2} \mathrm{CO}_{3}(99.8 \%), \mathrm{K}_{2} \mathrm{CO}_{3}(99 \%)$ and $\mathrm{Nb}_{2} \mathrm{O}_{5}(99.5 \%)$ were used as the raw materials with a molar ratio of 0.585:0.535:1.0. Amounts of excessive $\mathrm{Na}(17 \%)$ and $\mathrm{K}(7 \%)$ were determined based on sintering experiments leading to a stoichiometric KNN 50/50 ceramic target. The mixed raw materials were ball-milled for $24 \mathrm{~h}$ using alcohol as the dispersion medium, dried, and calcined at $850^{\circ} \mathrm{C}$ for $6 \mathrm{~h}$ in air. Then the calcined powders were mixed with a binder of $5 \mathrm{wt} \%$ polyvinyl butyral (PVB) and pressed into pellets with a $60 \mathrm{~mm}$-diameter and an $8 \mathrm{~mm}$ thickness under a uniaxial pressing @ $10 \mathrm{MPa}$ in a stainless-steel die. These pellets were then sintered at $1040^{\circ} \mathrm{C}$ in air for $2 \mathrm{~h}$ after burning of the PVB binder at $650^{\circ} \mathrm{C}$ for $5 \mathrm{~h}^{64}$. To avoid the formation of $\mathrm{NaOH}$ and $\mathrm{KOH}$, the sintered target was kept in a vacuum cabinet.

\section{Deposition of the KNN film}

Single-crystalline substrates of (100) $\mathrm{SrTiO}_{3}$ (STO) as well as the $\mathrm{SrRuO}_{3}$ (SRO) ceramic target were provided by Anhui Institute of Optics and Fine Mechanics (Chinese Academy of Sciences, China). A base pressure of $2.0 \times$ $10^{-4} \mathrm{~Pa}$ was achieved in a multi-target sputtering chamber prior to the sequential deposition of a bottom electrode (SRO) layer and a KNN film from the corresponding ceramic targets. KNN films of $\sim 150$ and $\sim 500 \mathrm{~nm}$ thicknesses with a SRO layer of a fixed thickness $\sim 60 \mathrm{~nm}$ were sputterdeposited in a mixed $\mathrm{Ar} / \mathrm{O}_{2}$ atmosphere (1.2 Pa, $\mathrm{Ar} / \mathrm{O}_{2}$ flow ratio=3:1). During the sputtering process, the substrate temperature was held at $600^{\circ} \mathrm{C}$. Right after their depositions, the KNN thin film heterostructures were kept at $600^{\circ} \mathrm{C}$ in $2.5 \mathrm{~Pa}$ pure oxygen for $20 \mathrm{~min}$, to help reduce the oxygen vacancies. Metal-ferroelectric-metal (MFM) testing structures were formed after deposition of top electrodes (circular Au pads with $\phi=$ $200 \mu \mathrm{m}$ ), which were sputtered at room temperature via a shadow mask.

\section{Characterization}

The phase structures and crystallographic orientations of the KNN films were analyzed using standard X-ray $2 \theta$-scans (in a commercial Rigaku Dmax-2500PC, Japan) and pole figure (R-156 Axis Spider, Smart Lab Rigaku, Japan; $40 \mathrm{kV}, 200 \mathrm{~mA}$ ). In the X-ray pole figure measurement, the (110) KNN peak was used. The $500 \mathrm{~nm}$-thick film sample was rotated by varying the tilt angle $\psi$ from $0^{\circ}$ to $90^{\circ}$, and the azimuthal angle $\varphi$ from $0^{\circ}$ to $360^{\circ}$ for every single $\psi$ value. Nanostructures of the films were analyzed using transmission electron microscopy (TEM, JEOL JEM-2100HR) with crosssectional samples prepared by using a focus ion beam (FIB) milling instrument (Scios2, FEI, USA). The surface morphology of the films was investigated by using an atomic force microscope (AFM, Micro Nano D-5A, with CSC21-AIBS AFM tips from Mikro Masch). The chemical bonding states of $\mathrm{K}, \mathrm{Na}, \mathrm{Nb}$, and $\mathrm{O}$ were analyzed using $\mathrm{X}$-ray photoelectron spectroscopy (XPS, Thermo Scientific ESCALAB 250Xi). The ferroelectric polarizationelectric field $(\mathrm{P}-\mathrm{E})$ hysteresis loops were measured by using a Radiant Precision LC ferroelectric tester (Radiant Technology, USA). The capacitance (C)-voltage (V) (C-V) tests, which were used to extract the dielectric permittivity $X$-electric field $E(X-E)$ curves, were carried out in a TH2828S LCR bridge (Tong hui Electronics, China), by superimposing a small AC signal $(\mathrm{V} p-\mathrm{p}=1 \mathrm{~V} @ 1 \mathrm{kHz})$ on a DC bias voltage sweeping from its negative maximum to its positive maximum, and vice versa.

\section{DATA AVAILABILITY}

The authors declare that the main data supporting the findings of this study are available within the paper. Other relevant data are available from the corresponding author upon reasonable request.
Received: 16 October 2020; Accepted: 23 March 2021; Published online: 06 May 2021

\section{REFERENCES}

1. Cole, M. W., Nothwang, W. D., Hubbard, C., Ngo, E. \& Ervin, M. Low dielectric loss and enhanced tunability of $\mathrm{Ba}_{0.6} \mathrm{Sr}_{0.4} \mathrm{TiO}_{3}$ based thin films via material compositional design and optimized film processing methods. J. Appl. Phys. 93, 9218-9225 (2003).

2. Tagantsev, A. K., Sherman, V. O., Astafiev, K. F., Venkatesh, J. \& Setter, N. Ferroelectric materials for microwave tunable applications. J. Electroceram. 11, 5-66 (2003).

3. Bao, P., Jackson, T. J., Wang, X. \& Lancaster, M. J. Barium strontium titanate thin film varactors for room-temperature microwave device applications. J. Phys. D: Appl. Phys. 41, 63001 (2008).

4. Zhao, J. et al. Effects of $\mathrm{Bi}_{2} \mathrm{O}_{3}, \mathrm{Sm}_{2} \mathrm{O}_{3}$ content on the structure, dielectric properties and dielectric tunability of $\mathrm{BaTiO}_{3}$ ceramics. J. Mater. Sci: Mater. Electron. 30, 19279-19288 (2019).

5. Everhardt, A. S. et al. Temperature-independent giant dielectric response in transitional $\mathrm{BaTiO}_{3}$ thin films. Appl. Phys. Rev. 7, 011402 (2020).

6. Zhang, W., Hu, F., Zhang, H. \& Ouyang, J. Investigation of the electrical properties of RF sputtered $\mathrm{BaTiO}_{3}$ films grown on various substrates. Mater. Res. Bull. 95, 23-29 (2017).

7. Aymen, S., Mascot, M., Jomni, F. \& Carru, J. High tunability in lead-free $\mathrm{Ba}_{0.85} \mathrm{Sr}_{0.15} \mathrm{TiO}_{3}$ thick films for microwave tunable applications. Ceram. Int. 45, 23084-23088 (2019).

8. Wang, F. \& Ma, W. Phase stability and dielectric properties of (011) epitaxial $\left(\mathrm{Ba}_{0.6} \mathrm{Sr}_{0.4}\right) \mathrm{TiO}_{3}$ films. J. Appl. Phys. 125, 082528 (2019).

9. Yuan, Z. et al. Large dielectric tunability and microwave properties of $\mathrm{Mn}$-doped $(\mathrm{Ba}, \mathrm{Sr}) \mathrm{TiO}_{3}$ thin films. Appl. Phys. Lett. 87, 152901 (2005).

10. $\mathrm{Wu}, \mathrm{M}$., Li, X., Yu, S., Sun, Y. \& Dong, H. Dielectric properties of the bulk and interfacial layers in ferroelectric $\mathrm{BaZr}_{0.2} \mathrm{Ti}_{0.8} \mathrm{O}_{3}$ thin films. Ceram. Int. 45, 10917-10923 (2019).

11. Zhong, S. et al. Highly tunable and temperature insensitive multilayer barium strontium titanate films. Appl. Phys. Lett. 90, 092901 (2007).

12. Cole, M. W., Ngo, E., Hirsch, S., Okatan, M. B. \& Alpay, S. P. Dielectric properties of MgO-doped compositionally graded multilayer barium strontium titanate films. Appl. Phys. Lett. 92, 072906 (2008).

13. Garten, L. M. \& Trolier-McKinstry, S. The field induced $e_{31, f}$ piezoelectric and Rayleigh response in barium strontium titanate thin films. Appl. Phys. Lett. 105, 132905 (2014).

14. Tang, X. G., Chew, K. H. \& Chan, H. L. W. Diffuse phase transition and dielectric tunability of $\mathrm{Ba}\left(\mathrm{Zr}_{\mathrm{y}} \mathrm{Ti}_{1-\mathrm{y}}\right) \mathrm{O}_{3}$ relaxor ferroelectric ceramics. Acta Mater. 52, 5177-5183 (2004).

15. Mahmoud, A. E., Afify, A. S. \& Parashar, S. K. S. Dielectric, tunability, leakage current, and ferroelectric properties of $\left(\mathrm{K}_{0.45} \mathrm{Na}_{0.55}\right)_{0.95} \mathrm{Li}_{0.05} \mathrm{NbO}_{3}$ lead free piezoelectric. J. Mater. Sci: Mater. Electron. 30, 2659-2668 (2019).

16. Yu, Z., Ang, C., Guo, R. \& Bhalla, A. S. Dielectric properties and high tunability of Ba $\left(\mathrm{Ti}_{0.7} \mathrm{Zr}_{0.3}\right) \mathrm{O}_{3}$ ceramics under dc electric field. Appl. Phys. Lett. 81, 1285-1287 (2002).

17. Blomqvist, M., Koh, J., Khartsev, S., Grishin, A. \& Andréasson, J. High-performance epitaxial $\mathrm{Na}_{0.5} \mathrm{~K}_{0.5} \mathrm{NbO}_{3}$ thin films by magnetron sputtering. Appl. Phys. Lett. 81, 337-339 (2002).

18. Li, T., Wang, G., Li, K., Du, G. \& Chen, Y. Electrical properties of lead-free KNN films on SRO/STO by RF magnetron sputtering. Ceram. Int. 40, 1195-1198 (2014).

19. Li, T., Wang, G., Remiens, D. \& Dong, X. Characteristics of highly (001) oriented (K, $\mathrm{Na}) \mathrm{NbO}_{3}$ films grown on $\mathrm{LaNiO}_{3}$ bottom electrodes by RF magnetron sputtering. Ceram. Int. 39, 1359-1363 (2013).

20. Ren, P. et al. Large nonlinear dielectric behavior in $\mathrm{BaTi}_{1-x} \mathrm{Sn}_{x} \mathrm{O}_{3}$. Sci. Rep. 7, 6693 (2017).

21. Peng, B., Fan, H., Li, Q. \& Zhang, Q. Dielectric tunability properties of the $\mathrm{Pb}\left[\left(\mathrm{Mg}_{1 /}\right.\right.$ $\left.\left.{ }_{3} \mathrm{Nb}_{2 / 3}\right)_{1-x} \mathrm{Zr}_{x}\right]_{\mathrm{O}_{3}}$ ceramics. J. Alloy. Compd. 549, 283-287 (2013).

22. Fujii, l. et al. Fabrication of $(\mathrm{K}, \mathrm{Na}) \mathrm{NbO}_{3}$ films on $\mathrm{SrRuO}_{3} /(001) \mathrm{SrTiO}_{3}$ substrates by pulsed laser deposition. Jpn. J. Appl. Phys. 54, $10 \mathrm{NA} 13$ (2015).

23. Fujii, I., Yamauchi, T., Imai, T., Adachi, H. \& Wada, T. Ferroelectric properties of (Li,K, $\mathrm{Na}) \mathrm{NbO}_{3}$ epitaxial films fabricated by pulsed laser deposition. Jpn. J. Appl. Phys. 53, 09PA09 (2014).

24. Ito, Y. et al. Growth of epitaxial $(\mathrm{K}, \mathrm{Na}) \mathrm{NbO}_{3}$ films with various orientations by hydrothermal method and their properties. Jpn. J. Appl. Phys. 58, SLLB14 (2019).

25. Khartsev, S., Grishin, A., Andrèasson, J., Koh, J. \& Song, J. Comparative characteristics of $\mathrm{Na}_{0.5} \mathrm{~K}_{0.5} \mathrm{NbO}_{3}$ films on Pt by pulsed laser deposition and magnetron sputtering. Integr. Ferroelectr. 55, 769-779 (2010).

26. Chen, L., Nagarajan, V., Ramesh, R. \& Roytburd, A. L. Nonlinear electric field dependence of piezoresponse in epitaxial ferroelectric lead zirconate titanate thin films. J. Appl. Phys. 94, 5147-5152 (2003). 
27. Li, T. et al. Influence of LNO top electrodes on electrical properties of KNN/LNO thin films prepared by RF magnetron sputtering. J. Am. Ceram. Soc. 96, 787-790 (2012).

28. Li, G. et al. Effects of excess amount of $\mathrm{K}$ and $\mathrm{Na}$ on properties of $\left(\mathrm{K}_{0.48} \mathrm{Na}_{0.52}\right)$ $\mathrm{NbO}_{3}$ thin films. Ceram. Int. 38, S279-S281 (2012).

29. Bell, A. J. Phenomenologically derived electric field-temperature phase diagrams and piezoelectric coefficients for single crystal barium titanate under fields along different axes. J. Appl. Phys. 89, 3907-3914 (2001).

30. Huang, Y. H. et al. Thermodynamic and phase-field studies of phase transitions, domain structures, and switching for $\mathrm{Ba}\left(\mathrm{Zr}_{x} \mathrm{Ti}_{1-x}\right) \mathrm{O}_{3}$ solid solutions. Acta Mater. 186, 609-615 (2020).

31. Jiang, Q. \& Qiu, J. H. The thickness dependence of ferroelectric and magnetic properties in epitaxial $\mathrm{BiFeO}_{3}$ thin films. J. Appl. Phys. 99, 103901 (2006).

32. Winchester, B., Wu, P. \& Chen, L. Q. Phase-field simulation of domain structures in epitaxial $\mathrm{BiFeO}_{3}$ films on vicinal substrates. Appl. Phys. Lett. 99, 052903 (2011).

33. Zhang, J. X. et al. Computer simulation of ferroelectric domain structures in epitaxial $\mathrm{BiFeO}_{3}$ thin films. J. Appl. Phys. 103, 094111 (2008).

34. Mtebwa, M., Tagantsev, A. K. \& Setter, N. Effect of elastic compliances and higher order Landau coefficients on the phase diagram of single domain epitaxial $\mathrm{Pb}(\mathrm{Zr}$, $\mathrm{Ti}_{3} \mathrm{O}_{3}$ (PZT) thin films. Aip Adv. 4, 127150 (2014).

35. Zhou, M., Wang, J., Chen, L. \& Nan, C. Strain, temperature, and electric-field effects on the phase transition and piezoelectric responses of $\mathrm{K}_{0.5} \mathrm{Na}_{0.5} \mathrm{NbO}_{3}$ thin films. $J$. Appl. Phys. 123, 154106 (2018).

36. Zhao, X., Wang, J. \& Chen, L. A thermodynamic study of phase transitions and electrocaloric properties of $\mathrm{K}_{0.5} \mathrm{Na}_{0.5} \mathrm{NbO}_{3}$ single crystals. Appl. Phys. Lett. 116, 092902 (2020).

37. Liu, D., Bai, G. \& Gao, C. Phase diagrams classification based on machine learning and phenomenological investigation of physical properties in $\mathrm{K}_{1-}{ }_{x} \mathrm{Na}_{\times} \mathrm{NbO}_{3}$ thin films. J. Appl. Phys. 127, 154101 (2020).

38. Wang, J. J., Wu, P. P., Ma, X. Q. \& Chen, L. Q. Temperature-pressure phase diagram and ferroelectric properties of $\mathrm{BaTiO}_{3}$ single crystal based on a modified Landau potential. J. Appl. Phys. 108, 114105 (2010).

39. Qiu, J. H., Zhao, T. X., Chen, Z. H., Yuan, N. Y. \& Ding, J. N. Orientation dependence of phase diagrams and physical properties in epitaxial $\mathrm{Ba}_{0.6} \mathrm{Sr}_{0.4} \mathrm{TiO}_{3}$ films. Solid. State Commun. 272, 57-62 (2018).

40. Peng, J. et al. A thermodynamic potential for barium zirconate titanate solid solutions. Npj. Comput. Mater. 4, 1-9 (2018).

41. $\mathrm{Li}, \mathrm{H}$. et al. Dependence of dielectric properties on internal stresses in epitaxial barium strontium titanate thin films. Appl. Phys. Lett. 78, 2354-2356 (2001).

42. Kim, D. H., Lee, H. N., Biegalski, M. D. \& Christen, H. M. Effect of epitaxial strain on ferroelectric polarization in multiferroic $\mathrm{BiFeO}_{3}$ films. Appl. Phys. Lett. 92, 012911 (2008).

43. Luo, J. et al. Domain evolution and piezoelectric response across thermotropic phase boundary in (K,Na) $\mathrm{NbO}_{3}$-based epitaxial thin films. ACS Appl. Mater. Inter. 9 13315-13322 (2017).

44. Luo, J. et al. Monoclinic (K,Na) $\mathrm{NbO}_{3}$ ferroelectric phase in epitaxial films. Adv. Electron. Mater. 3, 1700226 (2017)

45. von Helden, L. et al. Huge impact of compressive strain on phase transition temperatures in epitaxial ferroelectric $\mathrm{K}_{\mathrm{x}} \mathrm{Na}_{1-\mathrm{x}} \mathrm{NbO}_{3}$ thin films. Appl. Phys. Lett. 114, 232905 (2019)

46. Shiraishi, T. et al. Structural and electrical characterization of hydrothermally deposited piezoelectric (K,Na)(Nb,Ta)O 3 thick films. J. Mater. Sci. 55, 8829-8842 (2020).

47. Blochl, P. E. Projector augmented-wave method. Phys. Rev. B: Condens Matter 50 17953-17979 (1994).

48. Kresse, G. \& Joubert, D. From ultrasoft pseudopotentials to the projector augmented-wave method. Phys. Rev. B 59, 1758-1775 (1999).

49. Perdew, J. P., Burke, K. \& Ernzerhof, M. Generalized gradient approximation made simple. Phys. Rev. Lett. 77, 3865-3868 (1996).

50. Wang, K. \& Li, J. F. Analysis of crystallographic evolution in $\left(\mathrm{Na}_{1} \mathrm{~K}\right) \mathrm{NbO}_{3}$-based lead-free piezoceramics by x-ray diffraction. Appl. Phys. Lett. 91, 262902 (2007).

51. Yu, Q., Zhu, F., Cheng, L., Wang, K. \& Li, J. Determination of crystallographic orientation of lead-free piezoelectric $(\mathrm{K}, \mathrm{Na}) \mathrm{NbO}_{3}$ epitaxial thin films grown on $\mathrm{SrTiO}_{3}(100)$ surfaces. Appl. Phys. Lett. 104, 102902 (2014).

52. Akmal, M. H. M., MWarikh, A. R. \& Ralib, A. A. M. Vibrational piezoelectric energy harvester's performance using PZT vs KNN. Mater. Res. Express 6, 115708 (2019).

53. Wang, L., Ren, W., Shi, P. \& Wu, X. Cobalt doping effects on structures and electrical properties of lead-free ferroelectric $\mathrm{K}_{0.5} \mathrm{Na}_{0.5} \mathrm{NbO}_{3}$ films. J. Alloy. Compd. 608, 202-206 (2014)

54. Wang, L., Ren, W., Shi, P. \& Wu, X. Structures, electrical properties, and leakage current behaviors of un-doped and Mn-doped lead-free ferroelectric $\mathrm{K}_{0.5} \mathrm{Na}_{0.5} \mathrm{NbO}_{3}$ films. J. Appl. Phys. 115, 034103 (2014).

55. Wang, L., Yao, K., Goh, P. C. \& Ren, W. Volatilization of alkali ions and effects of molecular weight of polyvinylpyrrolidone introduced in solution-derived ferroelectric $\mathrm{K}_{0.5} \mathrm{Na}_{0.5} \mathrm{NbO}_{3}$ films. J. Mater. Res. 24, 3516-3522 (2009).
56. Wang, L. et al. Effect of pyrolysis temperature on $\mathrm{K}_{0.5} \mathrm{Na}_{0.5} \mathrm{NbO}_{3}$ thick films derived from polyvinylpyrrolidone-modified chemical solution. J. Am. Ceram. Soc. 93, 3686-3690 (2010).

57. $\mathrm{Xu}, \mathrm{L}$. et al. Fabrication of the transparent ferroelectric heterostructures based on KNN-based lead-free films. J. Phys. D: Appl. Phys. 53, 415301 (2020).

58. Zhang, Y. et al. Strain engineered $\mathrm{CaBi}_{2} \mathrm{Nb}_{2} \mathrm{O}_{9}$ thin films with enhanced electrical properties. Acs Appl. Mater. Inter. 8, 16744-16751 (2016).

59. Niu, M. et al. Integration-friendly, chemically stoichiometric $\mathrm{BiFeO}_{3}$ films with a piezoelectric performance challenging that of PZT. Acs Appl. Mater. Inter. 12, 33899-33907 (2020).

60. Kizaki, Y., Noguchi, Y. \& Miyayama, M. Defect control for low leakage current in $\mathrm{K}_{0.5} \mathrm{Na}_{0.5} \mathrm{NbO}_{3}$ single crystals. Appl. Phys. Lett. 89, 142910 (2006).

61. Wang, $\mathrm{L}$. et al. Enhanced ferroelectric properties in $\mathrm{Mn}$-doped $\mathrm{K}_{0.5} \mathrm{Na}_{0.5} \mathrm{NbO}_{3}$ thin films derived from chemical solution deposition. Appl. Phys. Lett. 97, 072902 (2010).

62. Zhang, W. et al. Space-charge dominated epitaxial $\mathrm{BaTiO}_{3}$ heterostructures. Acto Mater. 85, 207-215 (2015).

63. Wang, X. et al. Growth and field dependent dielectric properties of epitaxial $\mathrm{Na}_{0.5} \mathrm{~K}_{0.5} \mathrm{NbO}_{3}$ thin films. Appl. Phys. Lett. 73, 927-929 (1998)

64. Huan, Y., Wang, X. \& Li, L. Displacement of Ta-O bonds near polymorphic phase transition in Li-, Ta-, and Sb-modified ( $\mathrm{K}, \mathrm{Na}) \mathrm{NbO}_{3}$ ceramics. Appl. Phys. Lett. 104, 242905 (2014).

\section{ACKNOWLEDGEMENTS}

The authors are deeply grateful for the financial support from the National Natural Science Foundation of China (NSFC) (Grant Nos. 51772175, 52072150, 51702119), the seed funding for top talents in Qilu University of Technology (Shandong Academy of Sciences), the international cooperation research project of Qilu University of Technology (QLUTGJHZ2018003). Y.H. acknowledges the Young Elite Scientists Sponsorship Program by CAST. H.C. acknowledges the support from the Jiangsu Province NSFC (Grant No. BK20180764). J.O. would also like to acknowledge the support from the Independent Innovation Foundation of Shandong University (Grant Nos. 2018JC045, 2017ZD008, and 2015JC034).

\section{AUTHOR CONTRIBUTIONS}

L.H., Y.H., and J.O. designed the whole study. L.H. performed the research, collected and analyzed the data, and wrote the draft. Y.Y. and W.R. carried out the DFT study. J.O. analyzed and discussed the data, and revised the manuscript. Y.H. helped prepare the KNN target. H.C., Y.-Y.Z., Y.W., and J.Y. helped collect experimental data and participated in the discussion.

\section{COMPETING INTERESTS}

The authors declare no competing interests.

\section{ADDITIONAL INFORMATION}

Correspondence and requests for materials should be addressed to Y.Y., Y.H. or J.O.

Reprints and permission information is available at http://www.nature.com/ reprints

Publisher's note Springer Nature remains neutral with regard to jurisdictional claims in published maps and institutional affiliations.

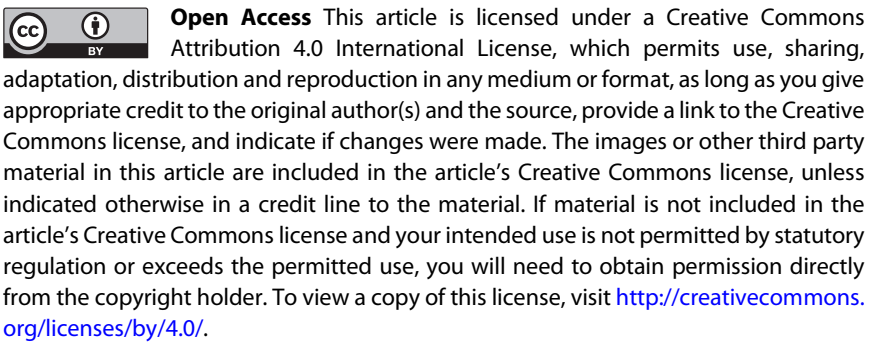

C) The Author(s) 2021 